\title{
Immune-related adverse event profile of combination treatment of PD-(L)1 checkpoint inhibitors and bevacizumab in non-small cell lung cancer patients: data from the FDA adverse event reporting system
}

\author{
Shuai Bai ${ }^{1,2}$, Tiantian Tian ${ }^{1,2}$, Jose M. Pacheco ${ }^{3}$, Motoko Tachihara ${ }^{4}$, Pingping Hu ${ }^{1,2}$, Jiandong Zhang ${ }^{1,2}$ \\ ${ }^{1}$ Department of Oncology, The First Affiliated Hospital of Shandong First Medical University \& Shandong Provincial Qianfoshan Hospital, Jinan, \\ China; ${ }^{2}$ Shandong Lung Cancer Institute, Jinan, China; ${ }^{3}$ Division of Medical Oncology, Department of Internal Medicine, University of Colorado \\ Cancer Center, Aurora, CO, USA; ${ }^{4}$ Division of Respiratory Medicine, Department of Internal Medicine, Kobe University Graduate School of \\ Medicine, Kobe, Japan \\ Contributions: (I) Conception and design: J Zhang, P Hu, S Bai; (II) Administrative support: T Tian; (III) Provision of study materials or patients: P \\ Hu, T Tian; (IV) Collection and assembly of data: S Bai, T Tian, JM Pacheco, M Tachihara; (V) Data analysis and interpretation: S Bai, T Tian, JM \\ Pacheco, M Tachihara; (VI) Manuscript writing: All authors; (VII) Final approval of manuscript: All authors. \\ Correspondence to: Pingping Hu; Jiandong Zhang. Department of Oncology, The First Affiliated Hospital of Shandong First Medical University \& \\ Shandong Provincial Qianfoshan Hospital, 16766 Jingshi Road, Jinan 250014, China. Email: hupingping0915@126.com; Jiandongzhang165@163.com.
}

Background: Immune checkpoint inhibitors (ICIs) and bevacizumab-based therapy are a promising treatment approach to significantly improving overall survival (OS) of non-small cell lung cancer (NSCLC) patients. However, the incidence of adverse events induced by a combination treatment with programmed cell death-1 or programmed death ligand 1 [PD-(L)1] inhibitor and bevacizumab remains unknown. The current evidence from prospective studies is limited. Thus, efforts using real-world data to further improve our understanding of the potential adverse events will be necessary.

Methods: The present study included 15,872 participants with NSCLC in the FDA Adverse Event Reporting System (FAERS) database from April 2013 to September 2019. The definition of adverse events (AEs) relied on the Medical Dictionary for Regulatory Activities (MedDRA). Statistical analysis was performed, and odds ratio (ORs) with 95\% confidence intervals (CIs) were calculated.

Results: Of the 15,872 participants with NSCLC, 15,463 cases were treated with the PD-(L)1 inhibitor monotherapy, while 409 cases were treated with both PD-(L)1 inhibitor and bevacizumab. Compared with monotherapy, combination therapy had lower risks of pneumonitis, respiratory failure, edema, disease progression, and death; however, combination therapy was also associated with significantly higher risks of pyrexia, general physical health deterioration, stomatitis, dehydration, thrombocytopenia, peripheral neuropathy, nephritis, bone marrow failure, immune thrombocytopenic purpura, neutropenia, and serious AEs. The results of the multivariate analysis suggested that combination therapy was the independent risk factor for pyrexia, neutropenia, nephritis, ITP, and the independent protective factor for respiratory failure.

Conclusions: We observed that the spectrum and risk of irAEs differed widely between therapeutic regimens, and irAEs involved multiple organ systems both in monotherapy or combination therapy. Deepening our understanding of irAEs has a great clinical value for improving individualized clinical patient management and the safety of medication use.

Keywords: Immune-related adverse events (irAEs); non-small cell lung cancer (NSCLC); PD-1; PD-L1; bevacizumab

Submitted Mar 22, 2021. Accepted for publication Jun 23, 2021.

doi: $10.21037 /$ tlcr-21-464

View this article at: https://dx.doi.org/10.21037/tlcr-21-464 


\section{Introduction}

Non-small cell lung cancer (NSCLC) is the leading cause of cancer-related death in the world and has a poor prognosis (1). At present, treatments for NSCLC patients include resection, chemotherapy, radiotherapy, targeted therapy, and immunotherapy (1). Cancer immunotherapies have created a significant paradigm shift in the therapeutic landscape of NSCLC. The frequently used immunotherapeutic drugs are immune checkpoint inhibitors (ICIs), such as specific antibodies targeting programmed cell death protein 1 (PD-1) and its ligand, programmed death-ligand 1 (PD-L1).

PD-1, primarily expressed on activated $\mathrm{T}$ cells, transmits inhibitory signals to limit the activity of $\mathrm{T}$ cells upon binding to PD-L1which is widely expressed on tumor cells. This then promotes tumor immune escape $(2,3)$. The antibodies against PD-1/PD-L1 prevent their interaction, thus relieving this suppression and restoring the activity of $\mathrm{T}$ cells. ICIs have been shown to improve survival in advanced NSCLC. The extent of PD-L1 expression is the most promising biomarker to select patients who can benefit from PD-1/PD-L1 inhibitors. In two randomized phase III trials (CheckMate 017 and 057) (4,5), among the advanced NSCLC patients, nivolumab continued to demonstrate benefit to overall survival (OS) and progression-free survival (PFS) compared to docetaxel, with 5 -year survival rates of $13.4 \%$ vs. $2.6 \%$ and PFS rates $8.0 \%$ vs. $0 \%$, respectively. Preliminary recent results of phase II trials (SAKK 16/14) on primary resectable stage IIIA (N2) NSCLC patients have shown an objective response rate (ORR) of $44.8 \%$ in the chemotherapy stage compared with $59.7 \%$ in the neoadjuvant immune stage (6). The phase III trial CheckMate-816 showed promising results that the pathological complete response (pCR) was $24 \%$, compared with 2.2 percent in the chemotherapy alone arm. (7). A recent pooled analysis showed that chemotherapy plus immunotherapy may improve efficacy outcomes over immunotherapy alone (8). To date, both antibodies targeting PD-1 (nivolumab and pembrolizumab), and PDL1 (atezolizumab, durvalumab) have been approved by the US Food and Drug Administration (FDA). However, only approximately $20 \%$ of unselected patients with advanced NSCLC have durable responses to monotherapy with ICIs (9). Therefore, developing new therapeutic strategies to improve patient response rates, especially combination therapies, have become increasingly common.

Vascular endothelial growth factor (VEGF)-dependent angiogenesis plays a critical role in tumor growth and metastasis of NSCLC $(10,11)$. Bevacizumab, a recombinant humanized immunoglobulin ( $\mathrm{Ig}$ ) G1 monoclonal antibody targeting VEGF, was the first angiogenesis inhibitor approved by the US FDA for treating advanced NSCLC patients according to significant clinical benefit demonstrated from two phase III trials (ECOG4599 and AVAIL) (12-14). It has been further proposed that VEGF can attenuate the antitumor immune response through multiple mechanisms, including the inhibition of $\mathrm{T}$ cells function (15), the suppression of dendritic cells (DCs) differentiation and activation $(16,17)$, and the induction and proliferation of regulatory $\mathrm{T}$ cells (Tregs) (18) and myeloid-derived suppressor cells (MDSCs) $(19,20)$. These observations suggest a potentially synergistic effect of combining immunotherapy with anti-VEGF therapy by reprogramming the tumor immune microenvironment.

However, when combination therapy is considered for treatment, the clinical benefit and overlapping toxicities of agents must be carefully considered. ICIs have brought new hope to patients at the cost of unique side effects, known as immune-related adverse events (irAEs). irAEs affect most human organs, including the skin, bowels, liver, kidneys, endocrine tissues, and even the central nervous system, with varying frequencies and severities (21). The most common side effects of bevacizumab are hypertension, asymptomatic proteinuria, thromboembolic events, gastrointestinal perforation, and wound-healing complications (22). However, whether combination treatment may increase the risk of toxicity in NSCLC patients is unknown, when considering the frequency of irAEs is dependent on the agents used and the specific characteristics of patients. Herein, we performed a comparative study to investigate whether there was an association between irAEs and the combination of PD-(L)1 and bevacizumab, based on the FDA Adverse Event Reporting System (FAERS), a realworld database.

We present the following article in accordance with the STROBE reporting checklist (available at https://dx.doi. org/10.21037/tlcr-21-464).

\section{Methods}

\section{Data collection}

We extracted data for patients with NSCLC registered between April 2013 and September 2019 in the FDA Adverse Event Reporting System (FAERS). The patients had been previously de-identified by the FAERS database. 
Therefore, no institutional review board approval was needed for the study. AEs were coded according to system organ class (SOC) and preferred terms (PTs) using the Medical Dictionary for Regulatory Activities (MedDRA). Serious AEs mean that one or more of the following outcomes were documented in the report: death, hospitalization, life-threatening complications, disability, congenital anomaly, and/or other serious outcomes. The PD-(L)1 inhibitors in the study included pembrolizumab, nivolumab, durvalumab, atezolizumab. Subsequently, we identified patients with AEs. Comorbidity included diabetes mellitus, chronic obstructive pulmonary disease, myocardial infarction, dementia, hemiplegia, connective tissue disease, liver disease, congestive heart failure, peripheral vascular disease, cerebrovascular disease, peptic ulcer disease, moderate-to-severe chronic kidney disease, leukemia, malignant lymphoma, and acquired immune deficiency syndrome. The study was conducted in accordance with the Declaration of Helsinki (as revised in 2013).

\section{Statistical analysis}

Data manipulation and statistical analyses were performed using Microsoft Excel (2010, Microsoft Corp.) and SPSS software (version 21.0, IBM Corp.). Odds ratios (ORs) and $95 \%$ confidence intervals (CIs) were calculated using relevant $2 \times 2$ contingency tables. Adjusted ORs were analyzed by multivariable logistic regression. The variables considered in the models were combination therapy, comorbidity, disease progression, stage, and chemotherapy. Comparisons for which the $95 \%$ CIs of the corresponding ORs did not include 1 were considered as significantly different. Venn diagrams were visualized using TBtools (23).

\section{Results}

\section{Basic information of the patients}

We identified 15,872 NSCLC cases. Among them, 409 and 15,463 cases were treated with the PD-(L)1 inhibitor in combination with bevacizumab or PD-(L) 1 inhibitor monotherapy, respectively. In the combination therapy group, the median age was 65 years, the median weight was $60 \mathrm{~kg}$, and $61.12 \%$ were female, and 370 patients received chemotherapy. Meanwhile, in the monotherapy group, the median age was 68 years, the median weight was $64 \mathrm{~kg}$, and $62.92 \%$ were female (Table 1). The ORs of irAEs were summarized according to system organ class, which included general disorders, gastrointestinal disorder, lung disorder, renal and urinary disorders, nervous system disorders, metabolism and endocrine disorders, blood and cardiac disorders, and hepatobiliary disorders (Figure $1 A, B, C, D, E, F, G, H$ ). In addition to the irAEs discussed above, other normal tissues would also suffer from irAEs, such as eye, reproductive system, musculoskeletal and connective tissue. However, those irAEs subgroups had too few patients to be included in the analysis. Statistically significant results are shown in Figure 2. The types of irAEs differed markedly between the two regimens. AntiPD-(L)1 monotherapy had a wide distribution of fatal irAEs including pneumonitis $(\mathrm{n}=1,089)$, pyrexia $(\mathrm{n}=839)$, respiratory failure $(n=379)$, edema $(n=356)$, and neutropenia $(n=345)$. The most frequent combination therapy irAEs were neutropenia $(n=63)$, pyrexia $(n=34)$, pneumonitis $(n=18)$, thrombocytopenia $(n=16)$, and nephritis $(n=15)$. Notably, simultaneous irAEs occurred in some patients (Figure 3A).

\section{The associations of irAEs with different immunotherapy regimens}

The incidence of irAEs is different according to the therapy class. Compared with monotherapy, combination therapy had a lower risk of pneumonitis (OR, 0.61; 95\% CI, 0.380.98), respiratory failure (OR, $0.29,95 \% \mathrm{CI}, 0.09-0.91)$, and edema (OR, 0.31; 95\% CI, 0.10-0.98). However, pyrexia (OR, 1.58; 95\% CI, 1.11-2.26), general physical health deterioration (GPHD, OR, 1.90; 95\% CI, 1.03-3.51), stomatitis (OR, 2.41; 95\% CI, 1.12-5.20), dehydration (OR, 2.60; 95\% CI, 1.32-5.17), thrombocytopenia (OR, 2.75; 95\% CI, 1.64-4.60), peripheral neuropathy (OR, 3.06; 95\% CI, 1.54-6.10), nephritis (OR, 3.29; 95\% CI, 1.92-5.62), bone marrow failure (OR, 4.34; 95\% CI, 1.71-11.00), immune thrombocytopenic purpura (ITP, OR, 5.59; 95\% CI, 2.76-11.32), neutropenia (OR, 7.98 95\% CI, 5.98$10.65)$ all showed a significantly higher chance of occurring with combination therapy than with monotherapy. Furthermore, combination therapy showed a significantly higher risk of serious AEs (OR, 3.13; 95\% CI, 1.55-6.32) and a lower risk of disease progression (OR, 0.42; 95\% CI, $0.30-0.58)$ and death (OR, 0.48; 95\% CI, 0.37-0.62).

To confirm this observation, we conducted multivariable logistic regression analysis to adjust for confounders, such as combination therapy, comorbidity, disease progression, stage, and chemotherapy (Tables 2-6). The results of the multivariate analysis suggested that combination therapy 
Table 1 Characteristics of 15,872 NSCLC patients. Clinical characteristics of patients treated with the PD-(L)1 inhibitor in combination with bevacizumab, and PD-(L)1 inhibitor monotherapy

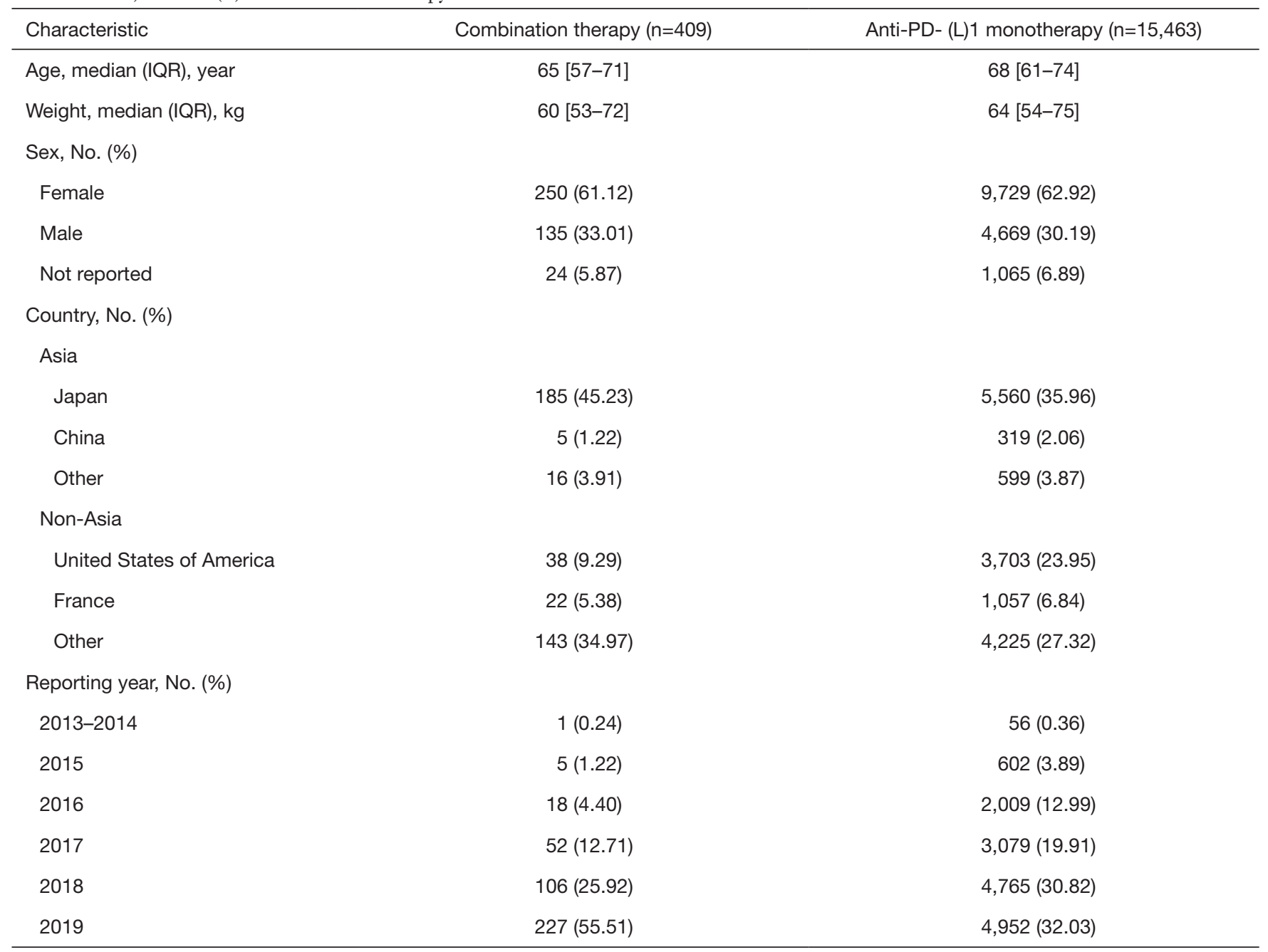

IQR, interquartile range.

was an independent risk factor for pyrexia (OR, 2.12; $95 \%$ CI, 1.43-3.16), neutropenia (OR, 1.89; 95\% CI, 1.39-2.56), nephritis (OR, 1.90; 95\% CI, 1.05-3.43), ITP (OR, 5.02; 95\% CI, 2.11-11.94). Meanwhile, combination therapy was found to be an independent protective factor for respiratory failure (OR, 0.27; 95\% CI, 0.09-0.86). The data were basically consistent with those of the univariate analysis. The results of the univariate analysis regarding comorbidity, disease progression, stage and chemotherapy can be seen in the supplementary materials (Figures S1-S4).

\section{Clinical characteristics of irAEs}

To determine the risk of irAEs associated death among
NSCLC patients, we assessed the fatality rates for different irAEs (Figure 3B). Patients with respiratory failure carried the highest risk of death, with 254 (66.49\%) deaths among 382 cases. General physical health deterioration, immune thrombocytopenic purpura, dehydration, pneumonitis, pyrexia, thrombocytopenia, edema, and stomatitis all had fatalities in $20 \%$ to $50 \%$ of reported cases.

\section{Discussion}

Combination treatment with the PD-(L)1 inhibitor and bevacizumab, as a new therapeutic modality, is currently the focus of research on the treatment of NSCLC. However, as treatment regimens move toward combination therapy, 
A General Disorders

$$
\text { irAEs }
$$

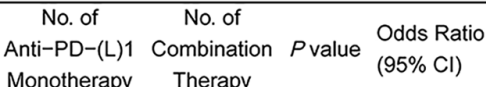

\begin{tabular}{lcc}
\hline Overall & & \\
Pyrexia & 839 & 34 \\
Pain & 849 & 22 \\
GPHD & 222 & 11 \\
Fatigue & 451 & 9 \\
Malaise & 289 & 8 \\
Asthen & 405 & 6 \\
Chilis & 89 & 6 \\
Edema & 359 & 3 \\
\hline
\end{tabular}

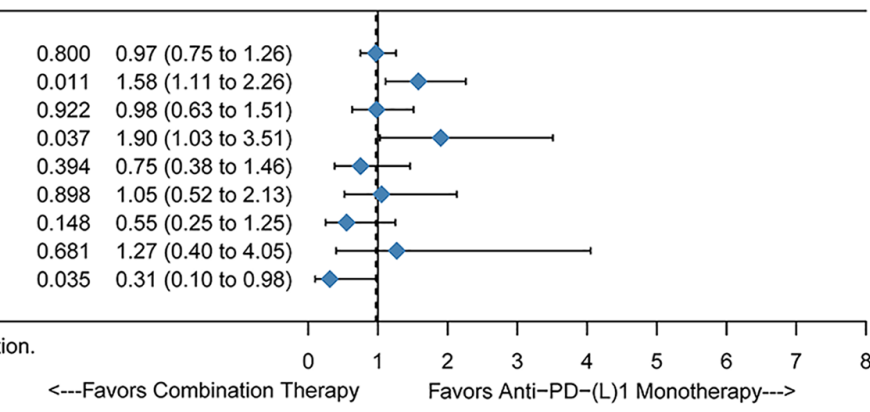

B Gastrointestinal Disorder

\begin{tabular}{lcc}
\hline irAEs & $\begin{array}{c}\text { No. of } \\
\text { Anti-PD-(L)1 } \\
\text { Monotherapy }\end{array}$ \\
\hline Com & Th \\
\hline Overall & \\
Diarroea & 680 \\
Colitis & 541 \\
Nausea & 403 \\
Vomiting & 296 \\
Abdominal Pain & 197 \\
Stomatitis & 111 \\
Constipation & 172 \\
Entrecolitits & 128 \\
Pancreatitis & 100 \\
Gastrititis & 51 \\
\hline
\end{tabular}

Pvalue Odds Ratio

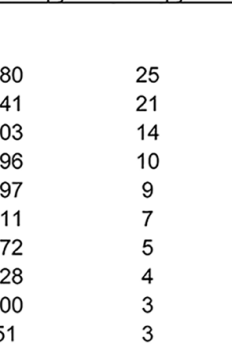
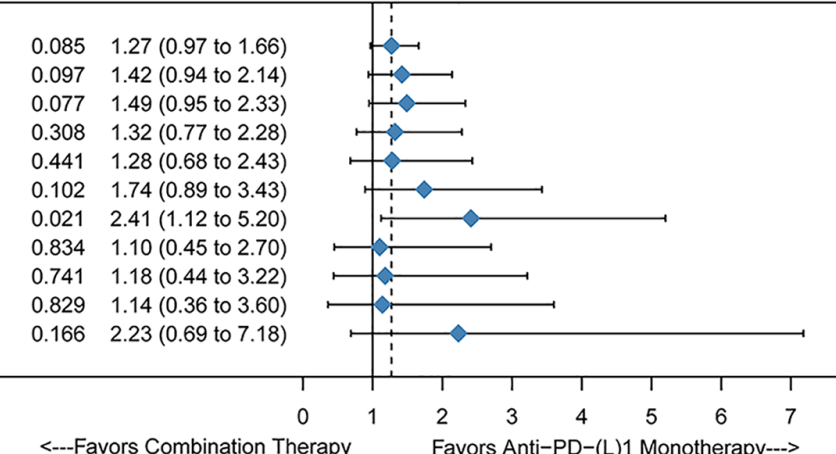

C Lung Disorder

Favors Combination The

$\begin{array}{cccccc}2 & 3 & 4 & 5 & 6 & 7 \\ \text { Favors Anti-PD-(L)1 } & \text { Monotherapy--a) }\end{array}$

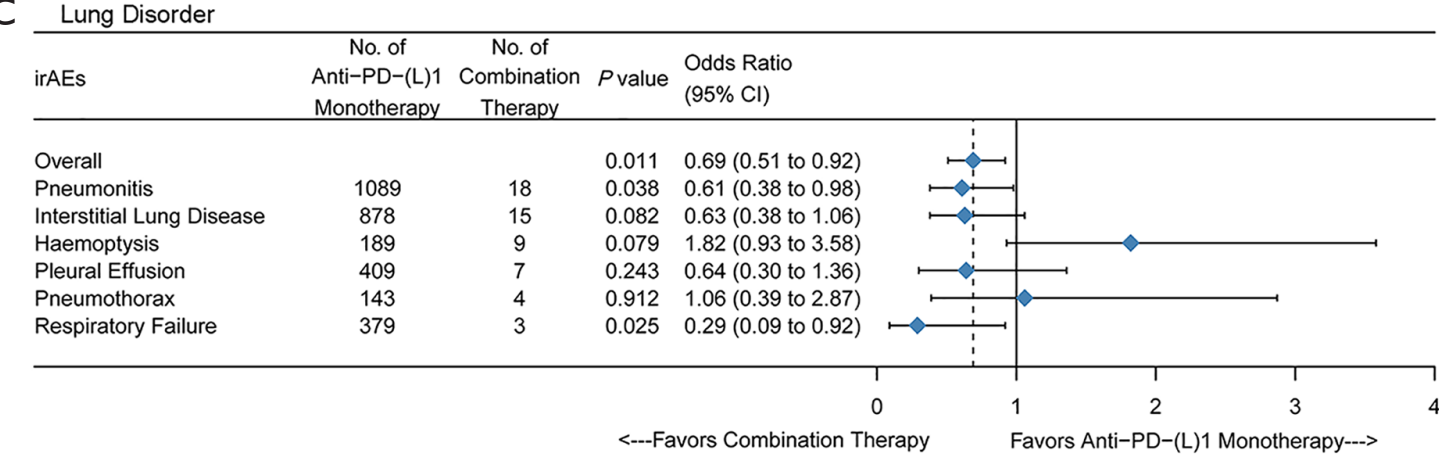

D Renal and Urinary Disorders

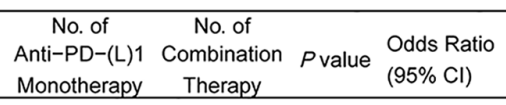

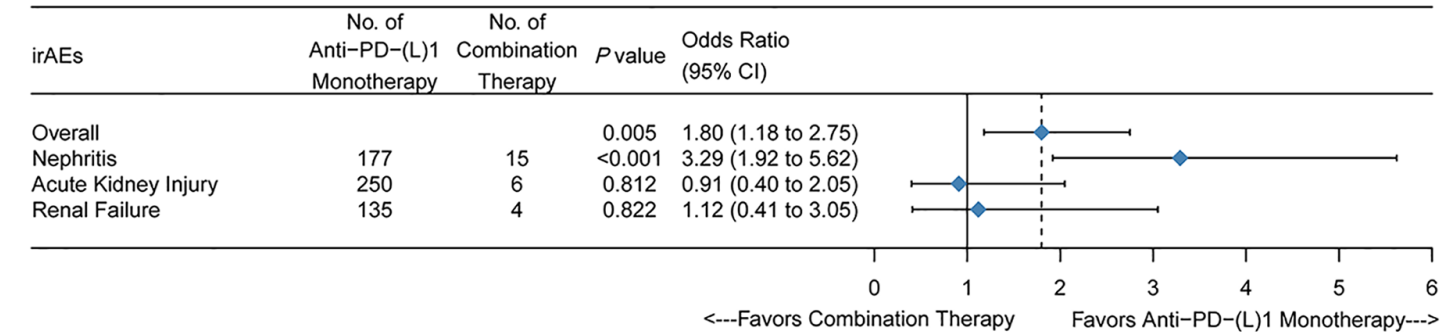

E Nervous System Disorders

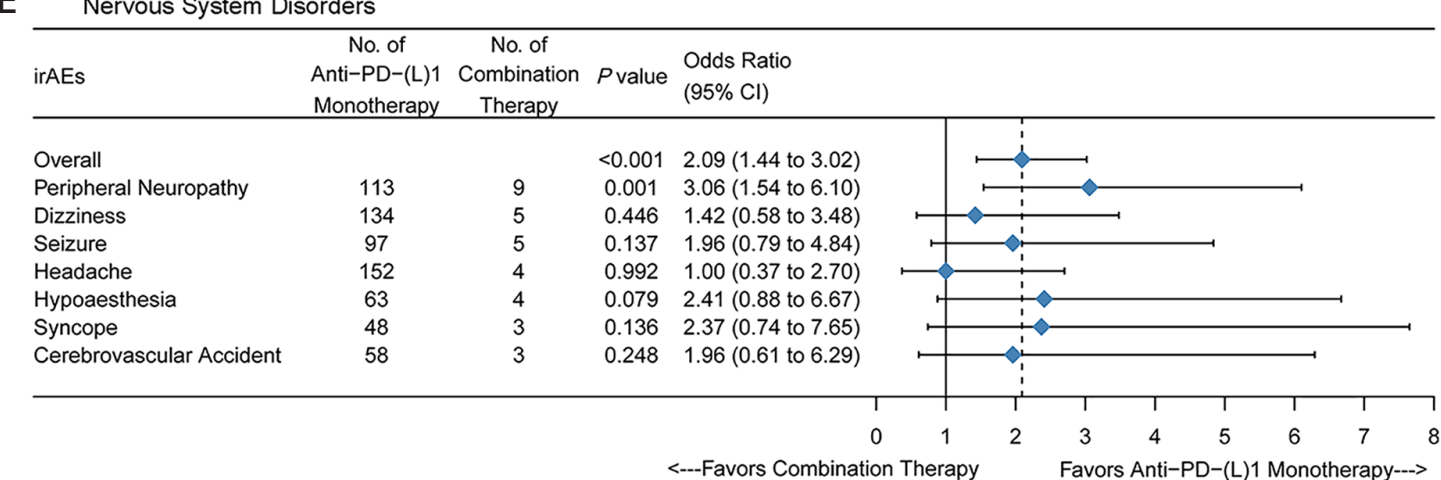

F Metabolism and Endocrine Disorders

2.-..Favors Combination Therapy

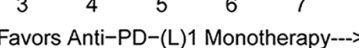

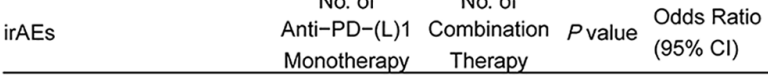

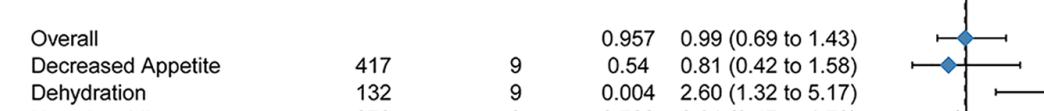

Diabetes Mellitus

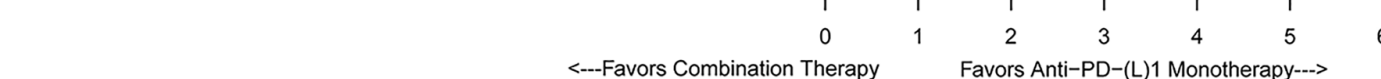

G Blood and cardiac disorders

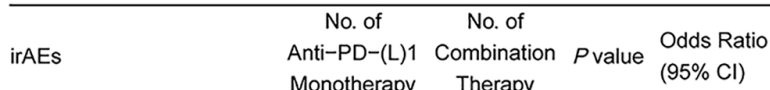

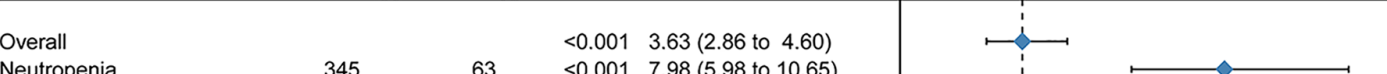

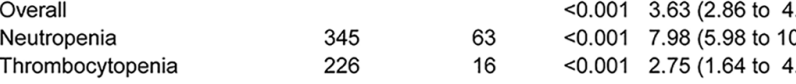

ITP

Bone Marrow Failure

396
62
44
82

Abbreviations: ITP, immune thrombocytopenic purpura,
Dilc, visseminated intravascular coagulatition.

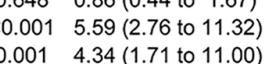

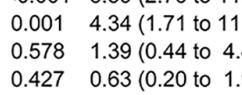

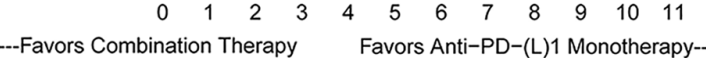

H Hepatobiliary Disorders

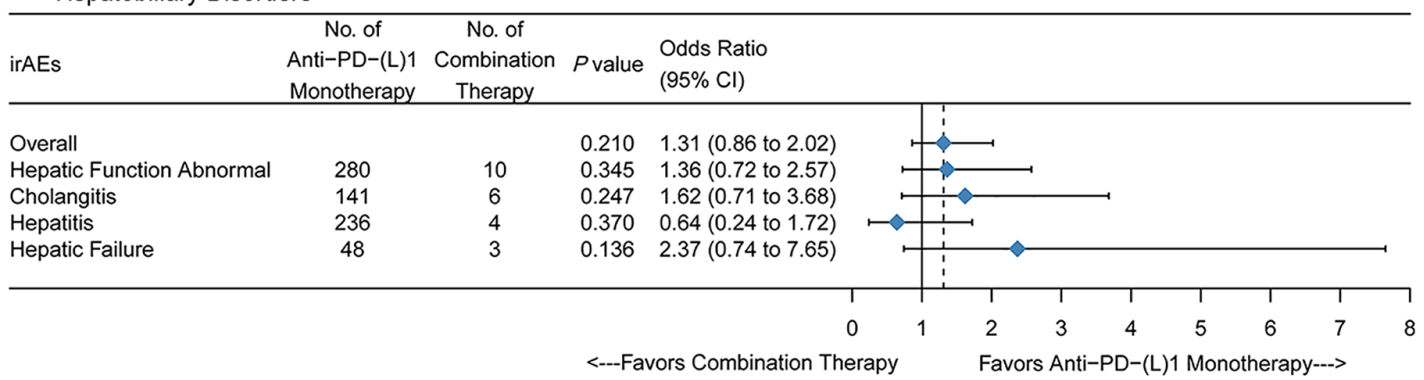

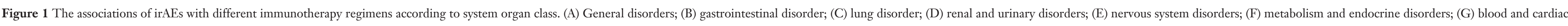

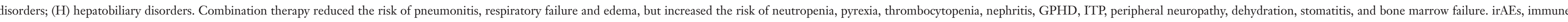
elated adverse events; GPHD, general physical health deterioration; ITP, immune thrombocytopenic purpura. 


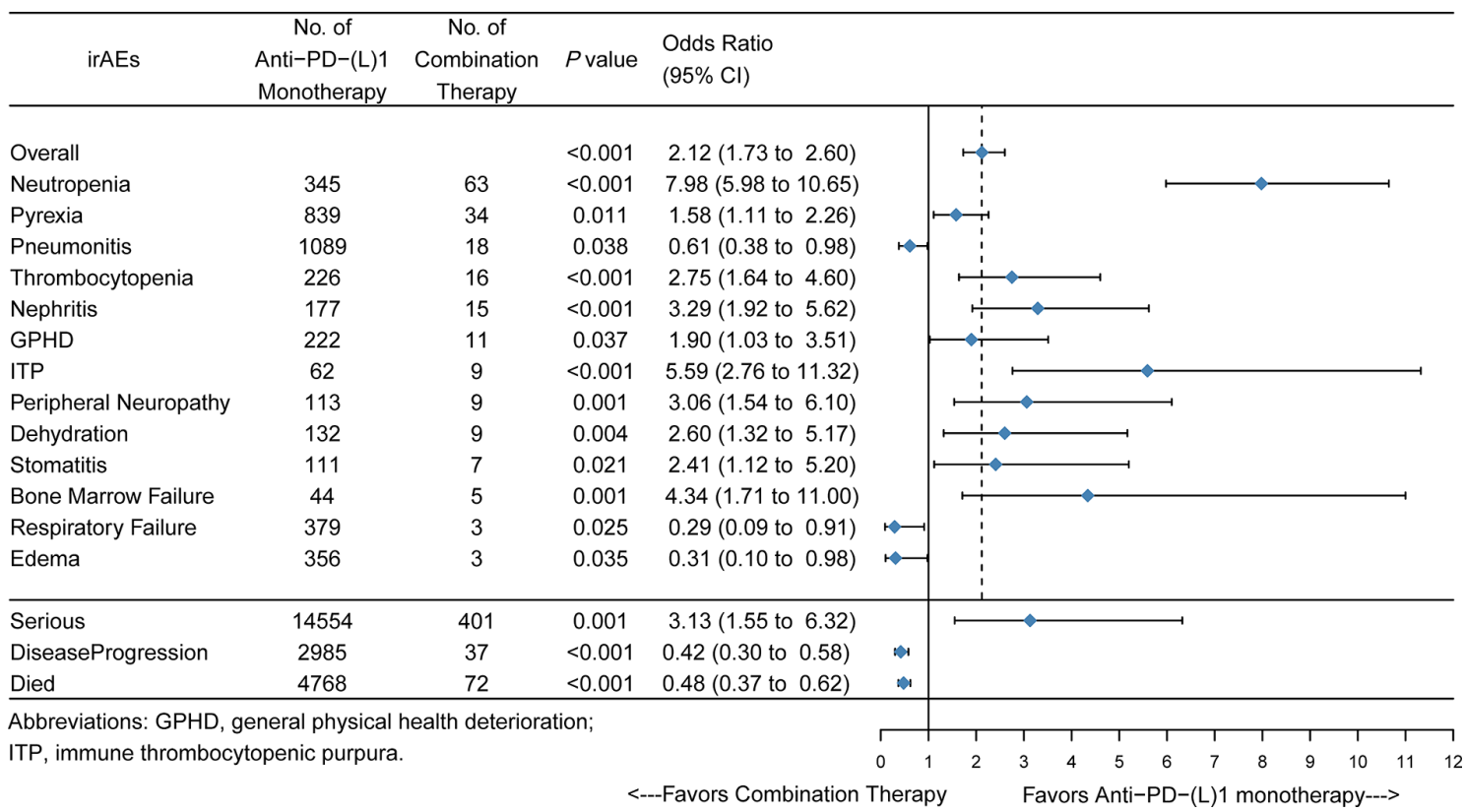

Figure 2 The associations of statistically significant irAEs with different immunotherapy regimens. Combination therapy increased overall irAE risk but reduced the risk of pneumonitis, respiratory failure, edema, death, and disease progression. irAEs, immune-related adverse events.
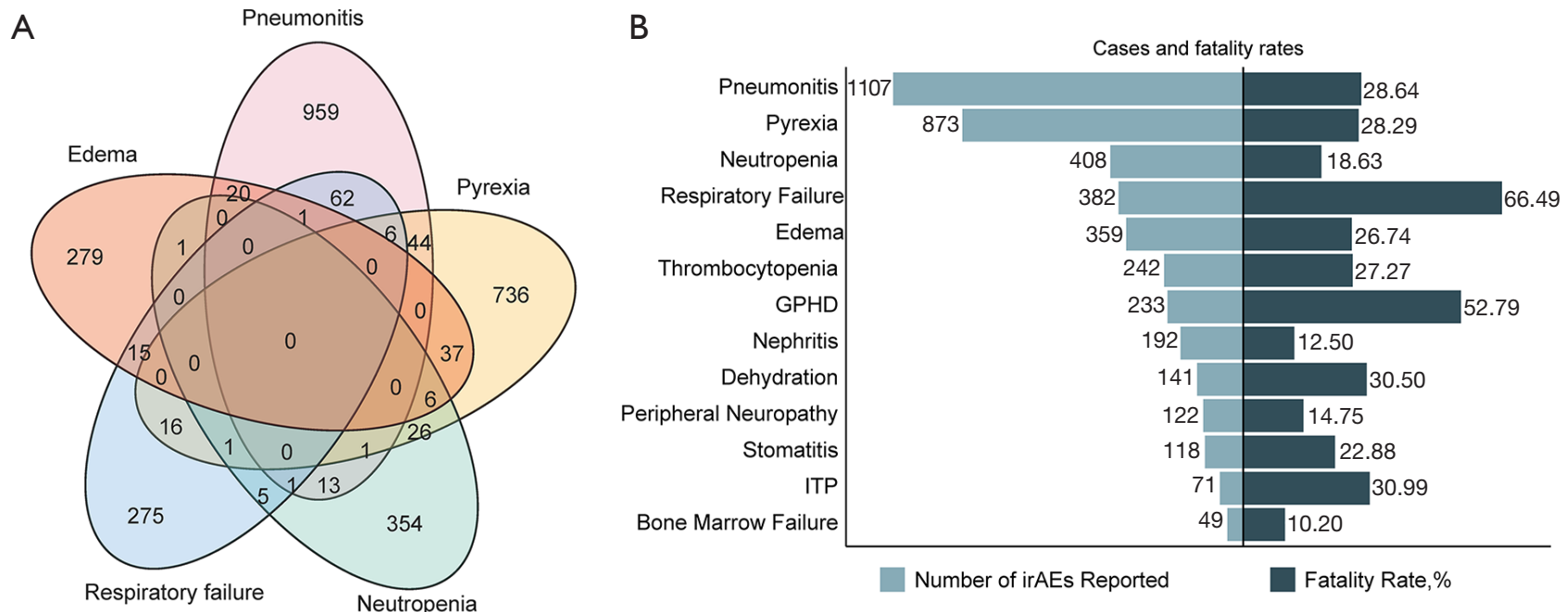

Figure 3 Clinical characteristics of irAEs. (A) Overlap of co-occurring fatal irAEs including neutropenia, pyrexia, pneumonitis, respiratory failure, edema. (B) Number of cases (left) and fatality rate (right) for each irAE. Note: the number of cases including combination treatment or PD-L1 inhibitor monotherapy. irAEs, immune-related adverse events; GPHD, general physical health deterioration; ITP, immune thrombocytopenic purpura.

one problem is becoming increasingly pressing: inevitable irAEs and unknown toxicity profiles. The current data are limited to small prospective studies, and a real-world study with a large sample size is still lacking. To our knowledge, the present study is the first large-scale real-world data analysis of this combination treatment in relation to adverse reactions based on the FAERS database. We found that the combination therapy reduced the risk of pneumonitis, 
Table 2 Multivariate analysis of risk factors of immune-related adverse events (irAEs). Combination therapy was an independent risk factor for pyrexia and neutropenia

\begin{tabular}{|c|c|c|c|c|c|c|}
\hline Variable & \multicolumn{2}{|l|}{ Pyrexia } & \multicolumn{2}{|l|}{ Neutropenia } & \multicolumn{2}{|c|}{ Pneumonitis } \\
\hline Combination therapy & $2.12(1.43-3.16)$ & $<0.001$ & 1.89 (1.39-2.56) & $<0.001$ & $0.76(0.46-1.24)$ & 0.270 \\
\hline Comorbidity & $0.48(0.32-0.71)$ & $<0.001$ & $0.79(0.67-1.43)$ & 0.913 & $1.04(0.80-1.34)$ & 0.789 \\
\hline Disease progression & $1.32(1.12-1.55)$ & 0.001 & $0.53(0.38-0.74)$ & $<0.001$ & $0.73(0.61-0.86)$ & $<0.001$ \\
\hline Chemotherapy & $0.74(0.59-0.92)$ & 0.007 & 9.09 (11.37-7.27) & $<0.001$ & $0.77(0.63-0.93)$ & 0.007 \\
\hline
\end{tabular}

$\mathrm{Cl}$, confidence interval.

Table 3 Multivariate analysis of risk factors of immune-related adverse events (irAEs). Combination therapy was an independent risk factor for nephritis

\begin{tabular}{|c|c|c|c|c|c|c|}
\hline Variable & \multicolumn{2}{|c|}{ Thrombocytopenia } & \multicolumn{2}{|l|}{ Nephritis } & \multicolumn{2}{|l|}{ GPHD } \\
\hline Combination therapy & $1.49(0.85-2.59)$ & 0.164 & $1.90(1.05-3.43)$ & 0.034 & $1.88(0.96-3.68)$ & 0.066 \\
\hline Comorbidity & $0.71(0.39-1.28)$ & 0.256 & $0.66(0.35-1.26)$ & 0.210 & $0.93(0.52-1.67)$ & 0.798 \\
\hline Disease progression & $0.78(0.54-1.11)$ & 0.164 & $0.32(0.18-0.56)$ & $<0.001$ & $2.26(1.72-2.98)$ & $<0.001$ \\
\hline Chemotherapy & 2.25 (1.66-3.04) & $<0.001$ & $1.65(1.15-2.36)$ & 0.006 & $1.30(1.86-0.91)$ & 0.154 \\
\hline
\end{tabular}

$\mathrm{Cl}$, confidence interval; GPHD, general physical health deterioration.

Table 4 Multivariate analysis of risk factors of immune-related adverse events (irAEs). Combination therapy was an independent risk factor for immune thrombocytopenic purpura

\begin{tabular}{|c|c|c|c|c|c|c|}
\hline Variable & \multicolumn{2}{|l|}{ ITP } & \multicolumn{2}{|c|}{ Peripheral neuropathy } & \multicolumn{2}{|c|}{ Dehydration } \\
\hline Combination therapy & $5.02(2.11-11.94)$ & $<0.001$ & $1.66(0.79-3.50)$ & 0.181 & $1.69(0.80-3.57)$ & 0.166 \\
\hline Comorbidity & $0.64(0.20-2.04)$ & 0.448 & $0.83(0.38-1.79)$ & 0.627 & $1.28(0.70-2.35)$ & 0.420 \\
\hline Disease Progression & $0.85(0.33-2.18)$ & 0.376 & $0.83(0.51-1.36)$ & 0.456 & $1.07(0.70-1.63)$ & 0.762 \\
\hline Chemotherapy & $1.16(0.59-2.26)$ & 0.667 & $2.21(1.44-3.38)$ & $<0.001$ & 1.62 (1.06-2.47) & 0.024 \\
\hline
\end{tabular}

$\mathrm{Cl}$, confidence interval; ITP, immune thrombocytopenic purpura.

respiratory failure, edema, disease progression, and death, while increasing the risk of pyrexia, GPHD, stomatitis, dehydration, thrombocytopenia, peripheral neuropathy, nephritis, bone marrow failure, ITP, neutropenia, and serious AEs.

Pneumonitis caused by ICIs has attracted considerable attention due to its high discontinuation and mortality rates. The increasing application of ICIs in the treatment of NSCLC will undoubtedly result in an increased absolute burden and mortality of pneumonitis. Previous metaanalyses have reported the incidence of pneumonitis during PD-(L)1 inhibitor monotherapy to be approximately $3.1 \%$ to $4.1 \%$ in NSCLC $(24,25)$. Another meta-analysis found that pneumonitis was more common when NSCLC 
Table 5 Multivariate analysis of risk factors of immune-related adverse events (irAEs). Combination therapy was not the independent risk factor for stomatitis and bone marrow failure

\begin{tabular}{|c|c|c|c|c|}
\hline Variable & \multicolumn{2}{|c|}{ Stomatitis } & \multicolumn{2}{|c|}{ Bone marrow failure } \\
\hline Combination therapy & $1.82(0.75-3.98)$ & 0.203 & $1.05(0.40-2.73)$ & 0.926 \\
\hline Comorbidity & $0.94(0.46-2.15)$ & 0.987 & $0.81(0.25-2.70)$ & 0.734 \\
\hline Disease progression & $1.73(1.15-2.60)$ & 0.008 & $0.31(0.10-1.01)$ & 0.052 \\
\hline Chemotherapy & $1.83(1.16-2.87)$ & 0.009 & 10.25 (5.55-18.96) & $<0.001$ \\
\hline
\end{tabular}

$\mathrm{Cl}$, confidence interval.

Table 6 Multivariate analysis of risk factors of immune-related adverse events (irAEs). Combination therapy was the independent protective factor for respiratory failure

\begin{tabular}{lcccc}
\hline \multirow{2}{*}{ Variable } & \multicolumn{2}{c}{ Respiratory failure } & & Edema \\
\cline { 2 - 3 } \cline { 4 - 5 } Combination therapy & Adjusted OR $(95 \% \mathrm{Cl})$ & P value & Adjusted OR $(95 \% \mathrm{Cl})$ & $0.38(0.12-1.21)$ \\
Comorbidity & $0.27(0.09-0.86)$ & 0.045 & 0.100 & 0.362 \\
Disease progression & $1.79(1.28-2.51)$ & 0.02 & $1.80-1.83)$ & 0.022 \\
Stage IV & $1.19(0.93-1.52)$ & 0.040 & $1.03(0.67-1.60)$ & 0.883 \\
Chemotherapy & $1.40(0.98-2.00)$ & 0.372 & $0.81(0.59-1.13)$ & 0.215 \\
\hline
\end{tabular}

$\mathrm{Cl}$, confidence interval.

patients received $\mathrm{PD}-(\mathrm{L}) 1$ combined with (cytotoxic T-lymphocyte-associated protein 4) CTLA4 inhibitors (26). However, our data suggest that the risk of PD-(L)1 inhibitor-induced pneumonitis may be significantly reduced when in combination with bevacizumab treatment, although it is not independent risk factor. A previous study showed that bevacizumab efficiently diminishes bronchial inflammation via reducing the expression of VEGFR2, and IL-6 genes and enhancing the expression of IL-10 gene (27). Besides, it has been proposed that VEGF may reprogram the tumor immune microenvironment through multiple mechanisms (15). Other previous study has indicated that pneumonitis may be related to those $\mathrm{T}$ cells that are similar to tumor-infiltrating $\mathrm{T}$ cells in patients treated with a PD-1 inhibitor (either nivolumab or pembrolizumab) (28). However, clinical comparative analysis of NSCLC patients treated with the PD-(L)1 inhibitor in combination with bevacizumab versus $\mathrm{PD}-(\mathrm{L}) 1$ inhibitor monotherapy is still lacking. Thus, the results of this study may be a valuable insight for physicians, especially regarding patients treated with $\mathrm{PD}-(\mathrm{L}) 1$ inhibitor and bevacizumab. Pneumonitis is a potentially fatal irAE if not treated promptly. Therefore, early detection, early diagnosis, and standard pharmaceutical administration are the keys to improving the cure rate and reducing the mortality. Further study is needed to reveal the exact biological mechanism behind this clinical observation.

As irAEs, neutropenia and thrombocytopenia are rare. A meta-analysis that included 9,324 patients found that the incidence of neutropenia and thrombocytopenia during PD-(L)1 inhibitor therapy were $0.94 \%$ and $2.84 \%$, respectively (29). In our present study, the combination treatment with $\mathrm{PD}-(\mathrm{L}) 1$ inhibitor and bevacizumab significantly increased the risk of neutropenia and thrombocytopenia in comparison with PD-(L) 1 inhibitor monotherapy. However, combined treatment was not an independent risk factor for thrombocytopenia. There are no clinical data available concerning the neutropenia and thrombocytopenia in relation to this combination treatment. It should be noted that bevacizumab may increase the risk of hematologic AEs. The E1505 study suggested that neutropenia was more common for NSCLC patients treated with bevacizumab for 1 year 
with adjuvant chemotherapy (30). A phase III randomized trial (bevacizumab in glioblastoma) showed a higher incidence of thrombocytopenia versus placebo (34.1\% vs. $27.3 \%$ ) (31). In a previous case report, a 59-yearold man with adenocarcinoma of the colon developed thrombocytopenia after bevacizumab treatment (32). The meta-analysis by Kut et al. indicated that VEGF is mostly concentrated in the platelets in cancer patients (33), while in vitro experiments have suggested that VEGF exerts potentiating effects on platelet activation (34). Thus, it is theoretically possible that the interaction of bevacizumab with the platelet VEGF contribute to thrombocytopenia. Awareness of this potential complication is important and may have a significant impact on the choice of therapeutic strategies.

Nephritis is a rare irAEs associated with ICIs. The present findings indicate that combination therapy may be associated with a higher risk of nephritis compared with monotherapy. To our knowledge, no previous study has reported this association. A previous meta-analysis found that the nephritis incidence in 12,808 oncologic patients treated with anti-PD-(L)1 agent was less than $1 \%$, while in phase 1 (35) and phase 2 trials (36), acute kidney injury in the form of nephritis was reported, with an incidence as high as $6.7 \%$. The current literature on immunerelated nephritis is limited, and the mechanism behind this phenomenon remains unknown. Therefore, multicentre, and multidisciplinary studies are necessary to establish the optimal management of immune-related nephritis patients to optimize their cancer and kidney outcomes.

We observed that PD-(L)1 inhibitor combined with bevacizumab had a protective effect on disease progression and death of NSCLC patients but increased the risk of serious AEs. This suggests that combined therapy may provide a survival benefit to NSCLC patients but may concurrently increase the complexity and severity of AEs. According to the NCCN guidelines, patients are required to undergo clinical exam at each visit to detect any potential irAEs and assess treatment response. Periodic imaging should be performed if there were clinical indications. The routine hematological tests are repeat performed prior to each treatment or every 4 days during immunotherapy, and then in 6-12 days. The skin and cardiovascular system need periodic testing based on abnormal baseline or symptoms. Pulmonary status needs to repeat oxygen saturation tests based on symptoms. Laboratory tests should be obtained every 4-6 weeks during immunotherapy, and followup every 6-12 weeks after completion of treatment to assess for organ function of Thyroid, pituitary, or adrenal. Pancreatic and musculoskeletal status does not require routine monitoring if asymptomatic. Therefore, for patients with advanced NSCLC, the patient's comorbidity, general condition, and survival prospects should be considered carefully before selecting the optimum treatment. Besides, close observation of patients' condition to implement timely treatment adjustments is recommended.

\section{Limitation}

Our study of FAERS data involved several limitations. First, it is difficult to establish a causal relationship between combination treatment and AEs. Second, there were no data on PD-(L)1 levels, TMB levels, the specific treatment and the time of onset and death for patients in the database. Third, clinical efficacy is usually evaluated using ORR and disease control rate, but this information was not available in the FAERS database, which solely includes information regarding disease progression. Thus, we could only analyse the relationship between the risk of irAEs and disease progression. Furthermore, healthcare professional and consumer reporting are spontaneous and voluntary, potentially leading to reporting bias and underreporting. Also, the reporting of the association between combination treatment and AE risk may be confounded by comorbid conditions and concomitant drugs. Although the FAERS has a large sample size, the number of certain fatal irAEs reports was too small to include in this analysis. Therefore, the data cannot be interpreted as definitive evidence of a reaction, but merely as an indication of an increased risk with a particular drug.

\section{Conclusions}

In conclusion, we observed that irAEs involved multiple organ systems in both treatments, while the spectrum and risk of irAEs differed widely between the therapeutic regimens. The mechanism by which irAEs occur is unclear and has a complex implication for anticancer treatment strategies. Given this potential risk, the key points of this study are to improve the overall understanding of the irAE spectrum for clinicians, particularly with respect to fatal irAEs, such as pneumonitis, nephritis, etc. In clinical practice, physicians should pay more attention to the early signs of these AEs and be able to diagnose and treat them, and this may have significant implications for clinical practice and ongoing research. 


\section{Acknowledgments}

The authors appreciate the academic support from AME Lung Cancer Collaborative Group.

Funding: This work was supported by the National Natural Science Foundation of China (No. 81672974, 81602719, and 81803043).

\section{Footnote}

Reporting Checklist: The authors have completed the STROBE reporting checklist. Available at https://dx.doi. org/10.21037/tlcr-21-464

Conflict of Interest: All authors have completed the ICMJE uniform disclosure form (available at https://dx.doi. org/10.21037/tlcr-21-464). The authors declare that this work was supported by the National Natural Science Foundation of China (No. 81672974, 81602719, and $81803043)$, and that the research was conducted in the absence of any commercial or financial relationships that could be construed as a potential conflict of interest. The authors have no other conflicts of interest to declare.

Ethical Statement: The authors are accountable for all aspects of the work in ensuring that questions related to the accuracy or integrity of any part of the work are appropriately investigated and resolved. The study was conducted in accordance with the Declaration of Helsinki (as revised in 2013).

Open Access Statement: This is an Open Access article distributed in accordance with the Creative Commons Attribution-NonCommercial-NoDerivs 4.0 International License (CC BY-NC-ND 4.0), which permits the noncommercial replication and distribution of the article with the strict proviso that no changes or edits are made and the original work is properly cited (including links to both the formal publication through the relevant DOI and the license). See: https://creativecommons.org/licenses/by-nc-nd/4.0/.

\section{References}

1. Siegel RL, Miller KD, Jemal A. Cancer statistics, 2019. CA Cancer J Clin 2019;69:7-34.

2. Xu-Monette ZY, Zhou J, Young KH. PD-1 expression and clinical PD-1 blockade in B-cell lymphomas. Blood 2018;131:68-83.
3. Jin HT, Ahmed R, Okazaki T. Role of PD-1 in regulating T-cell immunity. Curr Top Microbiol Immunol 2011;350:17-37.

4. Brahmer J, Reckamp KL, Baas P, et al. Nivolumab versus Docetaxel in Advanced Squamous-Cell Non-Small-Cell Lung Cancer. N Engl J Med 2015;373:123-35.

5. Borghaei H, Paz-Ares L, Horn L, et al. Nivolumab versus Docetaxel in Advanced Nonsquamous Non-Small-Cell Lung Cancer. N Engl J Med 2015;373:1627-39.

6. Bai R, Li L, Chen X, et al. Neoadjuvant and adjuvant immunotherapy: opening new horizons for patients with early-stage non-small cell lung cancer. Front Oncol 2020;10:575472.

7. Waser NA, Adam A, Schweikert B, et al. 1243P Pathologic response as early endpoint for survival following neoadjuvant therapy (NEO-AT) in resectable non-small cell lung cancer (rNSCLC): Systematic literature review and meta-analysis. Ann Oncol 2020;31:S806.

8. Akinboro O. editor. Outcomes of anti-PD-(L1) therapy in combination with chemotherapy versus immunotherapy (IO) alone for first-line (1L) treatment of advanced nonsmall cell lung cancer (NSCLC) with PD-L1 score 1-49\%: FDA pooled analysis 2021; ASCO Annual Meeting: American Society of Clinical Oncology.

9. Garon EB, Rizvi NA, Hui R, et al. Pembrolizumab for the treatment of non-small-cell lung cancer. N Engl J Med 2015;372:2018-28.

10. D'Amore PA. Vascular endothelial cell growth factor-a: not just for endothelial cells anymore. Am J Pathol 2007;171:14-8.

11. Han H, Silverman JF, Santucci TS, et al. Vascular endothelial growth factor expression in stage I non-small cell lung cancer correlates with neoangiogenesis and a poor prognosis. Ann Surg Oncol 2001;8:72-9.

12. Sandler A, Gray R, Perry MC, et al. Paclitaxel-carboplatin alone or with bevacizumab for non-small-cell lung cancer. N Engl J Med 2006;355:2542-50.

13. Sandler A, Yi J, Dahlberg S, et al. Treatment outcomes by tumor histology in Eastern Cooperative Group Study E4599 of bevacizumab with paclitaxel/carboplatin for advanced non-small cell lung cancer. J Thorac Oncol 2010;5:1416-23.

14. Reck M, von Pawel J, Zatloukal P, et al. Phase III trial of cisplatin plus gemcitabine with either placebo or bevacizumab as first-line therapy for nonsquamous non-small-cell lung cancer: AVAil. J Clin Oncol 2009;27:1227-34.

15. Ziogas AC, Gavalas NG, Tsiatas M, et al. VEGF directly 
suppresses activation of $T$ cells from ovarian cancer patients and healthy individuals via VEGF receptor Type 2. Int J Cancer 2012;130:857-64.

16. Gabrilovich D, Ishida T, Oyama T, et al. Vascular endothelial growth factor inhibits the development of dendritic cells and dramatically affects the differentiation of multiple hematopoietic lineages in vivo. Blood 1998;92:4150-66.

17. Gabrilovich DI, Chen HL, Girgis KR, et al. Production of vascular endothelial growth factor by human tumors inhibits the functional maturation of dendritic cells. Nat Med 1996;2:1096-103.

18. Terme M, Pernot S, Marcheteau E, et al. VEGFAVEGFR pathway blockade inhibits tumor-induced regulatory T-cell proliferation in colorectal cancer. Cancer Res 2013;73:539-49.

19. Detmar M, Brown LF, Schon MP, et al. Increased microvascular density and enhanced leukocyte rolling and adhesion in the skin of VEGF transgenic mice. J Invest Dermatol 1998;111:1-6.

20. Ko JS, Zea AH, Rini BI, et al. Sunitinib mediates reversal of myeloid-derived suppressor cell accumulation in renal cell carcinoma patients. Clin Cancer Res 2009;15:2148-57.

21. De Velasco G, Je Y, Bosse D, et al. Comprehensive Metaanalysis of Key Immune-Related Adverse Events from CTLA-4 and PD-1/PD-L1 Inhibitors in Cancer Patients. Cancer Immunol Res 2017;5:312-8.

22. Kazazi-Hyseni F, Beijnen JH, Schellens JH. Bevacizumab. Oncologist 2010;15:819-25.

23. Chen C, Chen H, Zhang Y, et al. TBtools: An Integrative Toolkit Developed for Interactive Analyses of Big Biological Data. Mol Plant 2020;13:1194-202.

24. Ma K, Lu Y, Jiang S, et al. The relative risk and incidence of immune checkpoint inhibitors related pneumonitis in patients with advanced cancer: a meta-analysis. Front Pharmacol 2018;9:1430.

25. Nishino M, Giobbie-Hurder A, Hatabu H, et al. Incidence of programmed cell death 1 inhibitor-related pneumonitis in patients with advanced cancer: a systematic review and meta-analysis. JAMA Oncol 2016;2:1607-16.

26. Naidoo J, Wang X, Woo KM, et al. Pneumonitis in Patients Treated With Anti-Programmed Death-1/ Programmed Death Ligand 1 Therapy. J Clin Oncol 2017;35:709-17.

27. Bolandi SM, Abdolmaleki Z, Assarehzadegan MA. Bevacizumab regulates inflammatory cytokines and inhibits VEGFR2 signaling pathway in an ovalbumin-induced rat model of airway hypersensitivity. Inflammopharmacology
2021. [Epub ahead of print]. doi: 10.1007/s10787-02100798-8.

28. Laubli H, Koelzer VH, Matter MS, et al. The T cell repertoire in tumors overlaps with pulmonary inflammatory lesions in patients treated with checkpoint inhibitors. Oncoimmunology 2018;7:e1386362.

29. Petrelli F, Ardito R, Borgonovo K, et al. Haematological toxicities with immunotherapy in patients with cancer: a systematic review and meta-analysis. Eur J Cancer 2018;103:7-16.

30. Wakelee HA, Dahlberg SE, Keller SM, et al. Adjuvant chemotherapy with or without bevacizumab in patients with resected non-small-cell lung cancer (E1505): an openlabel, multicentre, randomised, phase 3 trial. Lancet Oncol 2017;18:1610-23.

31. Saran F, Chinot OL, Henriksson R, et al. Bevacizumab, temozolomide, and radiotherapy for newly diagnosed glioblastoma: comprehensive safety results during and after first-line therapy. Neuro Oncol 2016;18:991-1001.

32. Kumar J, Bhargava M, Aggarwal S. Bevacizumabinduced reversible thrombocytopenia in a patient with adenocarcinoma of colon: rare adverse effect of bevacizumab. Case Rep Oncol Med 2012;2012:695430.

33. Kut C, Mac Gabhann F, Popel AS. Where is VEGF in the body? A meta-analysis of VEGF distribution in cancer. $\mathrm{Br}$ J Cancer 2007;97:978-85.

34. Selheim F, Holmsen H, Vassbotn FS. Identification of functional VEGF receptors on human platelets. FEBS Lett 2002;512:107-10.

35. Robert C, Ribas A, Wolchok JD, et al. Anti-programmeddeath-receptor-1 treatment with pembrolizumab in ipilimumab-refractory advanced melanoma: a randomised dose-comparison cohort of a phase 1 trial. Lancet 2014;384:1109-17.

36. Ribas A, Puzanov I, Dummer R, et al. Pembrolizumab versus investigator-choice chemotherapy for ipilimumabrefractory melanoma (KEYNOTE-002): a randomised, controlled, phase 2 trial. Lancet Oncol 2015;16:908-18.

Cite this article as: Bai S, Tian T, Pacheco JM, Tachihara $\mathrm{M}$, Hu P, Zhang J. Immune-related adverse event profile of combination treatment of $\mathrm{PD}-(\mathrm{L}) 1$ checkpoint inhibitors and bevacizumab in non-small cell lung cancer patients: data from the FDA adverse event reporting system. Transl Lung Cancer Res 2021;10(6):2614-2624. doi: 10.21037/tlcr-21-464 


\section{Supplementary}

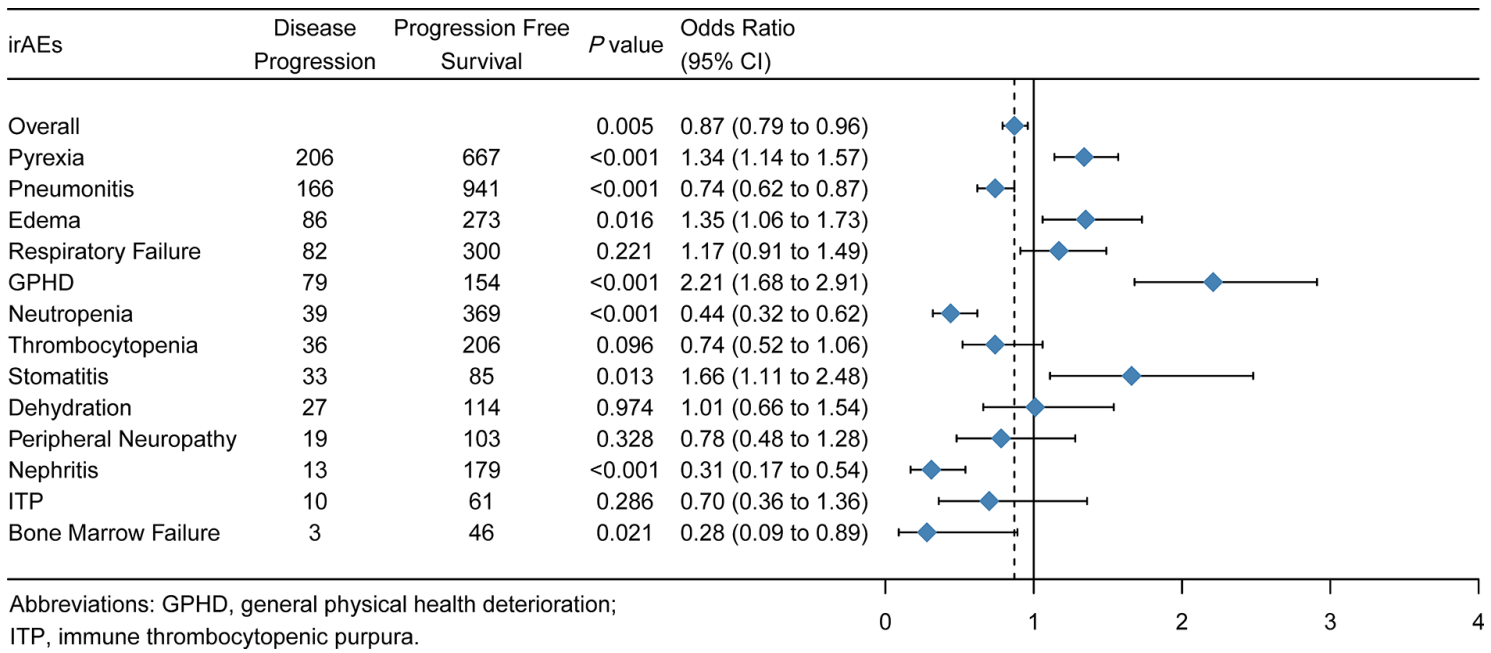

Figure S1 The associations of immune-related adverse events (irAEs) with disease progression. Patients with tumor progression had a lower risk of pneumonitis, neutropenia, nephritis, and bone marrow failure.

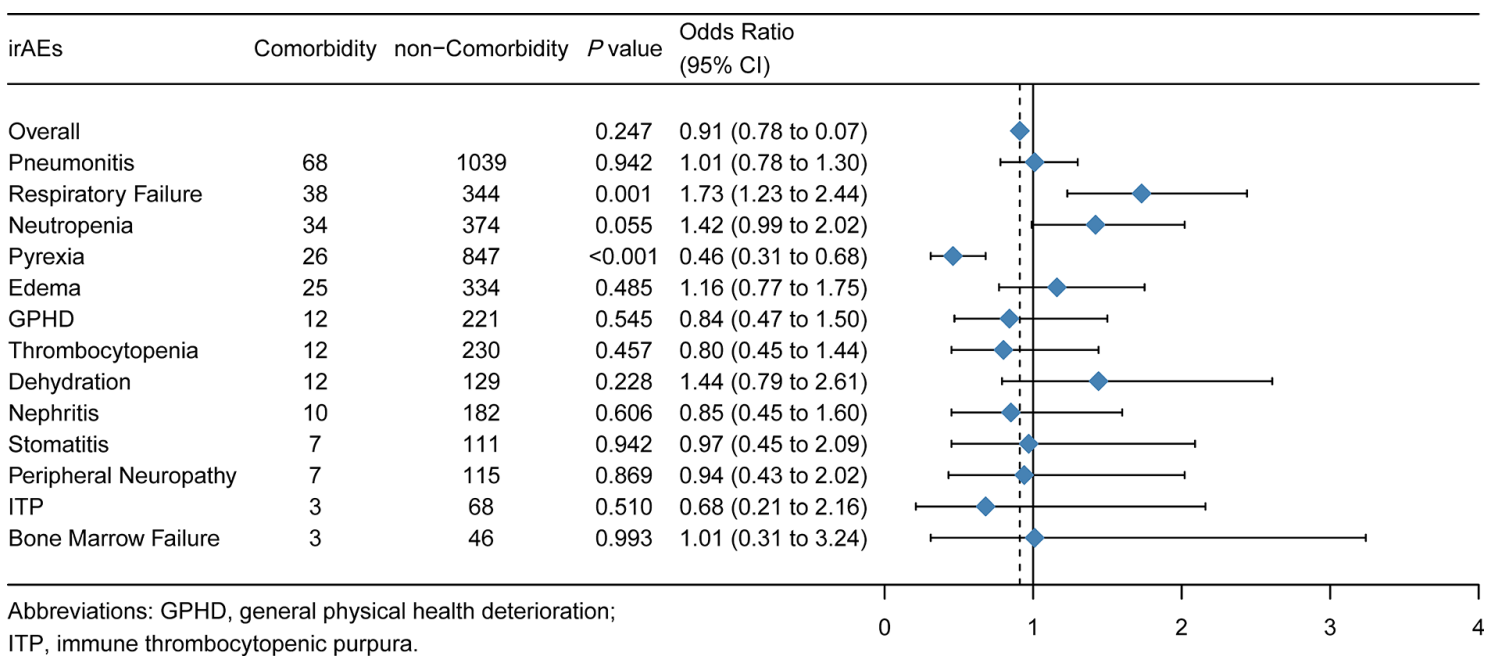

Figure S2 The associations of immune-related adverse events (irAEs) with comorbidity. Patients with comorbidity had lower risk of pyrexia. However, there was no statistical significance between comorbidity status with overall irAE risk. 


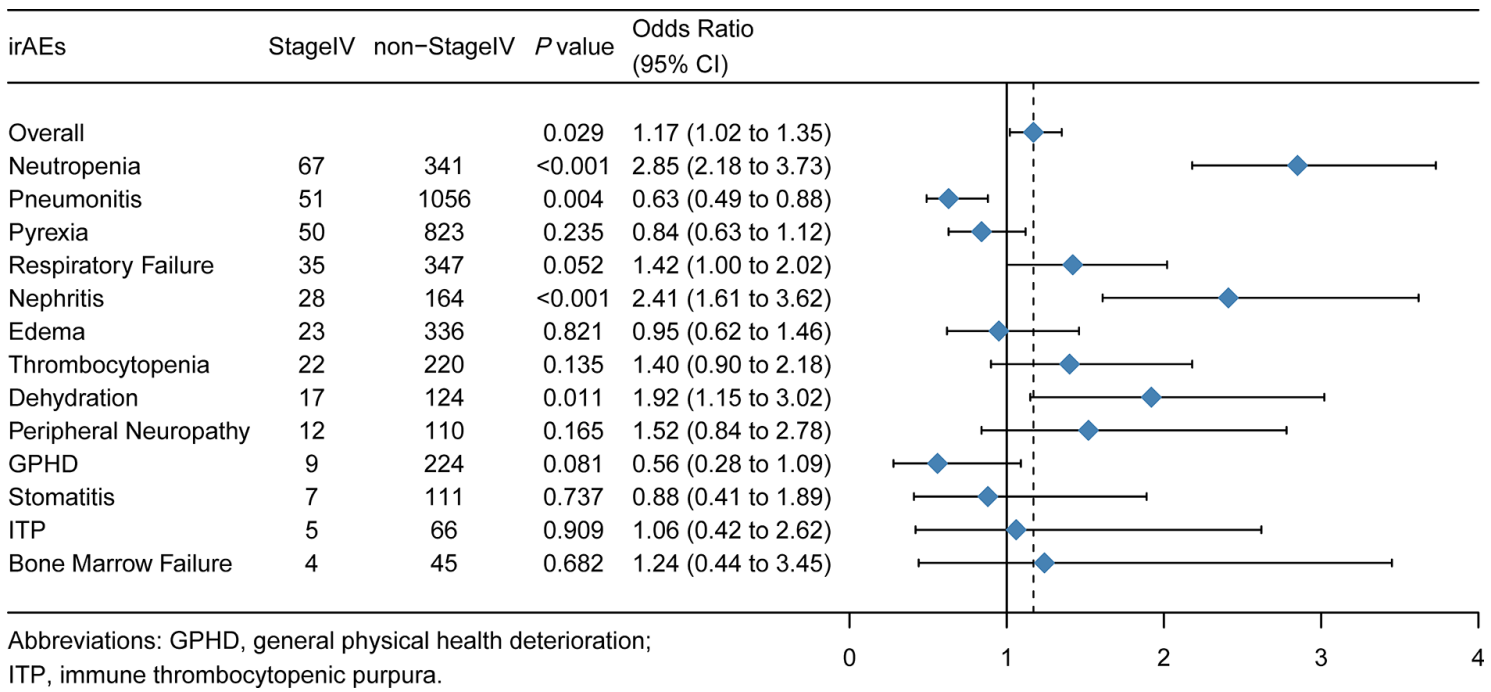

Figure S3 The associations of immune-related adverse events (irAEs) with stage. Patients with stage IV had increased overall irAE risk but reduced the risk of pneumonitis.

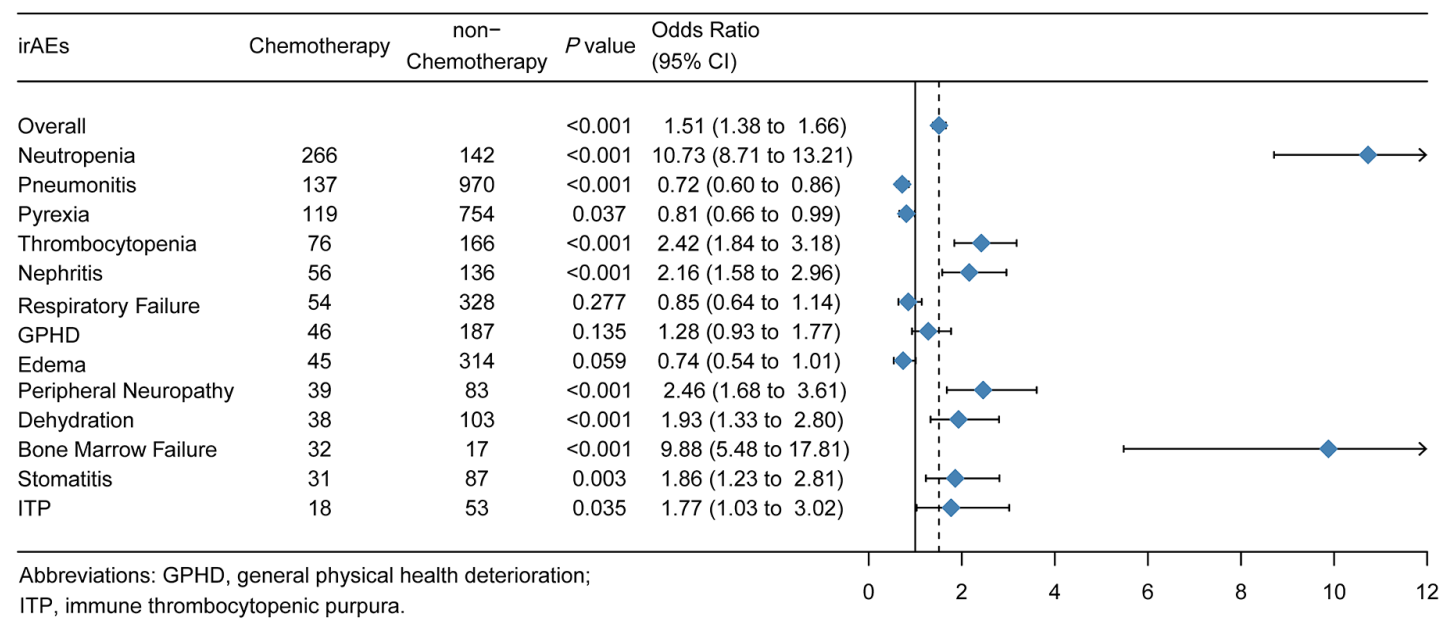

Figure S4 The associations of immune-related adverse events (irAEs) with chemotherapy. Patients with tumor progression had a higher risk of neutropenia, thrombocytopenia, nephritis, neuropathy peripheral, dehydration, bone marrow failure, stomatitis, ITP. 